\title{
Folliculite ankylostomienne compliquant une dépigmentation cutanée volontaire au Bénin
}

\author{
Hookworm-related folliculitis in a woman performing skin bleaching in Benin
}

\author{
B. Dégboé $\cdot$ C. Koudoukpo $\cdot$ N. Elégbédé $\cdot$ N. Agbéssi $\cdot$ A. Habib $\cdot$ C. d'Almeïda $\cdot$ P. Ngolo $\cdot$ F. Akpadjan $\cdot$ \\ H. Adégbidi · F. Atadokpèdé
}

Reçu le 5 juillet 2019; accepté le 9 décembre 2019

(C) Société de pathologie exotique et Lavoisier SAS 2019

Résumé La folliculite ankylostomienne est une forme folliculaire de larva migrans cutanée ankylostomienne rarement observée. Nous en rapportons un cas chez une patiente de 33 ans, se dépigmentant depuis 12 ans. Elle a consulté pour une éruption évoluant depuis une semaine, diffuse, très prurigineuse, apparue deux jours après le contact avec le sol d'un entrepôt souillé par des déjections de chats. L'examen physique retrouvait de nombreuses lésions de folliculites, érythémateuses coexistant avec des cordons serpigineux de dermatite rampante sur les zones d'extension des membres, le dos, les fesses et l'abdomen, typiques de folliculite ankylostomienne. Un traitement à base d'albendazole $400 \mathrm{mg}$ par jour pendant 7 jours a permis d'obtenir la guérison.

Mots clés Larva migrans cutanée · Ankylostome · Folliculite · Dépigmentation cutanée $\cdot$ Albendazole

Hôpital · Bénin · Afrique subsaharienne

Abstract Hookworm-related cutaneous larva migrans is
caused by the subcutaneous migration of hookworm larvae.
The characteristic sign is a serpiginous cutaneous track. Fol-
liculitis is an uncommon clinical manifestation. We report a
case of hookworm-related folliculitis in a woman performing
skin bleaching. A 33 -year-old woman presented with a
widespread cutaneous eruption made of erythematous and
itchy follicular papulo-nodules, with erythematous serpigi-
nous tracks, about 5-10cm long and numerous streaks of

B. Dégboé $(\bowtie) \cdot N$. Elégbédé · A. Habib · C. d'Almeïda

P. Ngolo · F. Akpadjan - H. Adégbidi · F. Atadokpèdé Clinique universitaire de dermatologie-vénérologie du Centre national hospitalier et universitaire de Cotonou, Faculté des sciences de la santé, Université d'Abomey-Calavi, Bénin e-mail : kebdegboe@yahoo.fr

C. Koudoukpo · N. Agbéssi

Service de dermatologie-vénérologie du Centre hospitalier universitaire départemental du Borgou-Alibori, Faculté de médecine, Université de Parakou, Bénin scratching, located on the limbs, buttocks and trunk. The cutaneous lesions occurred two days after contact with the floor of a warehouse contamined by faeces of cats. The lesions have been evolving for a week and have been treated with antibiotics without success. Clinical examination found cutaneous atrophy, purple stretch marks and hyperpigmentation of dorsal parts of fingers joints. The patient reported having been using bleaching cosmetics containing topical steroids and hydroquinone for twelve years. She was cured within 4 weeks with oral albendazole $400 \mathrm{mg}$ per day during 7 consecutive days.

Keywords Cutaneous larva migrans - Hookworms · Folliculitis · Skin bleaching · Albendazole $\cdot$ Hospital · Benin $\cdot$ Sub-Saharan Africa

\section{Introduction}

La larva migrans cutanée ankylostomienne (LMCA) est l'une des parasitoses cutanées fréquentes et endémiques dans les régions tropicales [4-6,14]. Elle est due à la pénétration transcutanée accidentelle et à la survie en impasse parasitaire chez l'homme de larves d'ankylostomes d'origine animale par les chiens et les chats $[4,7,13]$.

Classiquement, elle se présente comme une dermatite rampante sous forme de cordons sous-cutanés, érythématopapuleux, serpigineux, prurigineux et migrateurs $[4,6]$. Le terme de folliculite ankylostomienne a été utilisé pour la première fois par Miller en 1991 pour décrire des lésions de folliculites particulièrement prurigineuses dues à des larves d'ankylostomes d'origine animale [10].

La folliculite ankylostomienne est une forme atypique et rare de la LMCA. Elle est le plus souvent rapportée chez des touristes à « peau claire », ayant séjourné dans un pays d'endémie $[1,2,6,10]$. Elle serait rarement observée chez le sujet noir, même dans les régions endémiques $[4,14]$. Nous 
rapportons un cas de folliculite ankylostomienne sur un terrain de dépigmentation artificielle.

\section{Observation}

Une patiente de 33 ans a consulté pour une éruption papuleuse très prurigineuse associée à des lésions sinueuses évoluant depuis une semaine. Le prurit était sans horaire particulier. Elle aurait été traitée par des antibiotiques dont la flucloxacilline, sans succès. Cette symptomatologie serait survenue deux jours après le contact avec le sol d'un entrepôt souillé par des déjections de chats. L'examen physique montrait de nombreuses lésions de folliculites, indolores, érythémateuses dont certaines étaient excoriées, le tout disposé de façon éparse sur les zones d'extension des membres, le dos, les fesses et l'abdomen (Fig. 1 et 2). Ces lésions coexistaient aux membres avec des cordons serpigineux érythémateux, d'environ $5-10 \mathrm{~cm}$, sinueux (Fig. 3) et de nombreuses stries de grattage. Il n'y avait pas d'atteinte des espaces interdigitaux et des poignets. Le reste de l'examen physique notait des signes de dépigmentation volontaire à type d'atrophie cutanée, de vergetures pourpres et d'une hyperpigmentation cutanée des articulations inter phalangiennes. La patiente a déclaré utiliser des cosmétiques dépig- mentants à base de dermocorticoïdes et d'hydroquinone depuis douze ans.

Le diagnostic de folliculite ankylostomienne diffuse sur un terrain de dépigmentation cosmétique volontaire a été évoqué sur la base des éléments anamnestiques et physiques. Un traitement oral à base d'albendazole $400 \mathrm{mg} / \mathrm{jour}$ pendant 7 jours a permis d'observer une disparition progressive du prurit au bout de deux semaines et une disparition totale des lésions cutanées en 4 semaines (Fig. 4) : la tolérance a été bonne.

\section{Discussion}

Deux points de notre observation suscitent l'intérêt : la durée d'incubation très courte ( 2 jours) et les difficultés diagnostiques dans ce contexte de dépigmentation cutanée.

La forme folliculaire de la larva migrans cutanée ankylostomienne, dite folliculite ankylostomienne, est rarement observée en pays tropicaux [12,16,18,19]. Après la description du cas princeps en Australie en 1991 [10], quelques cas de folliculite ankylostomienne ont été rapportés [1$4,13,18,19]$. Sa fréquence par rapport aux séries de LMCA est passée de 2/69 cas en 1995 dans une étude prospective réalisée chez des patients de retour de voyage des régions

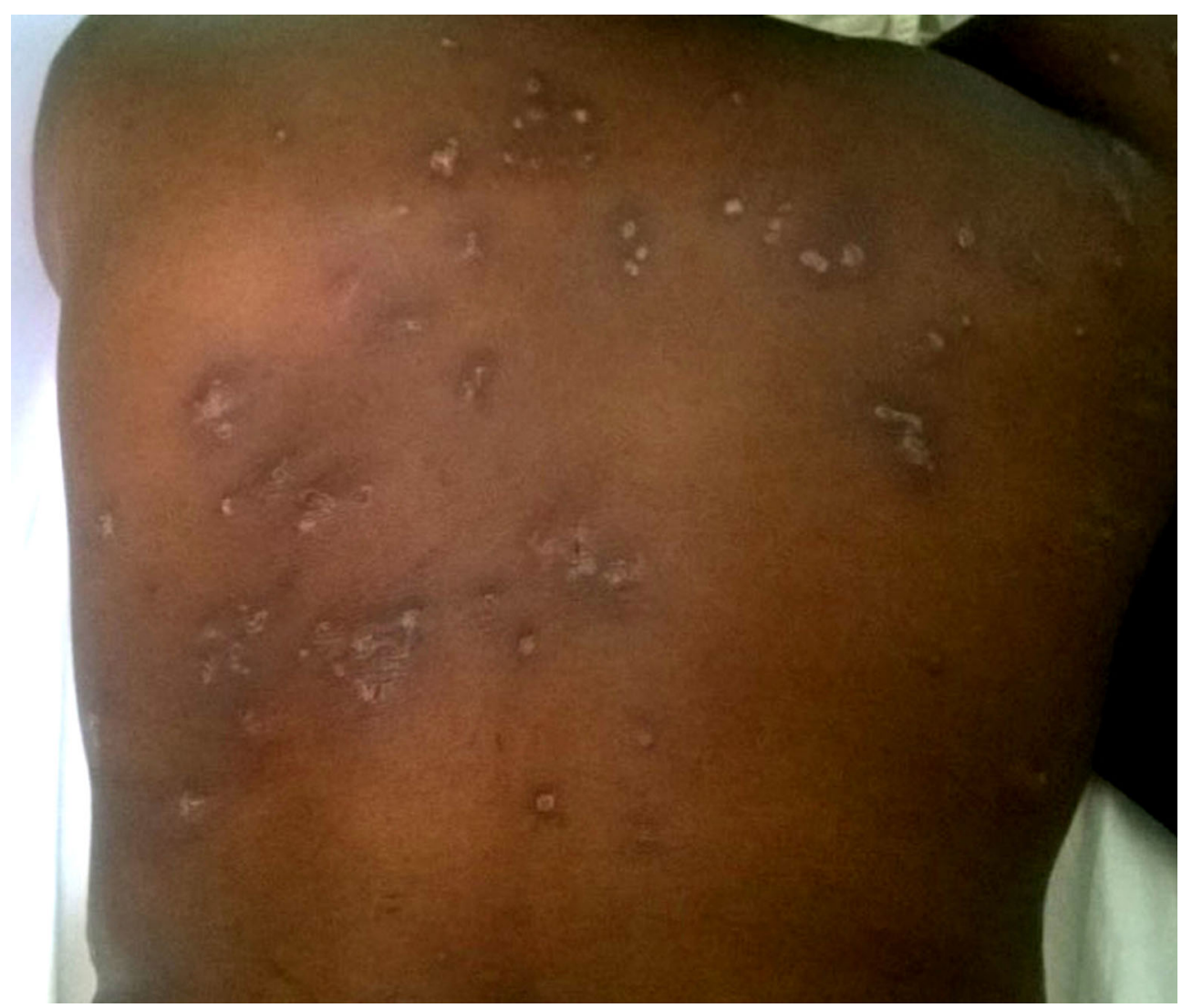

Fig.1 Nombreux papulo-nodules folliculaires du dos / Many follicular papulo-nodules of the back 


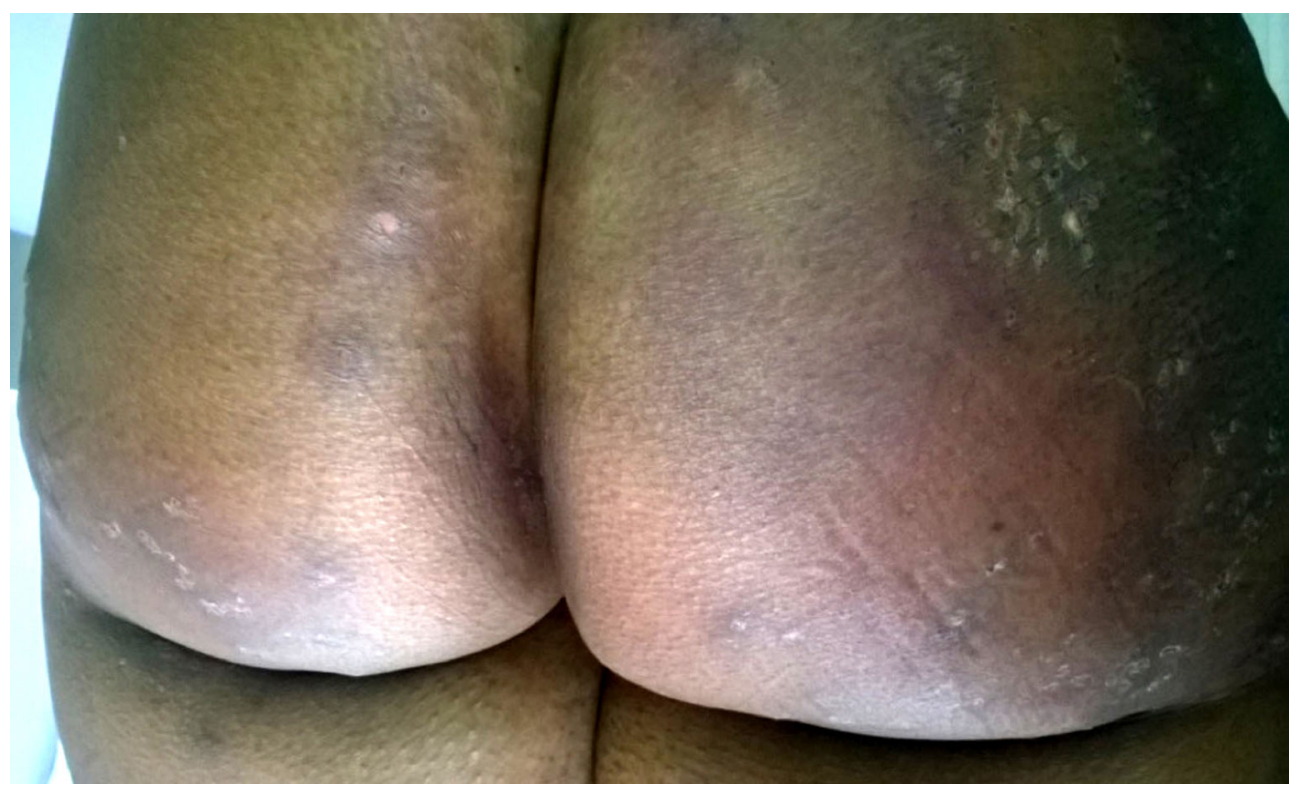

Fig. 2 Nombreux papulo-nodules folliculaires excoriés des fesses / Numerous follicular excoriated papulo-nodules of buttocks

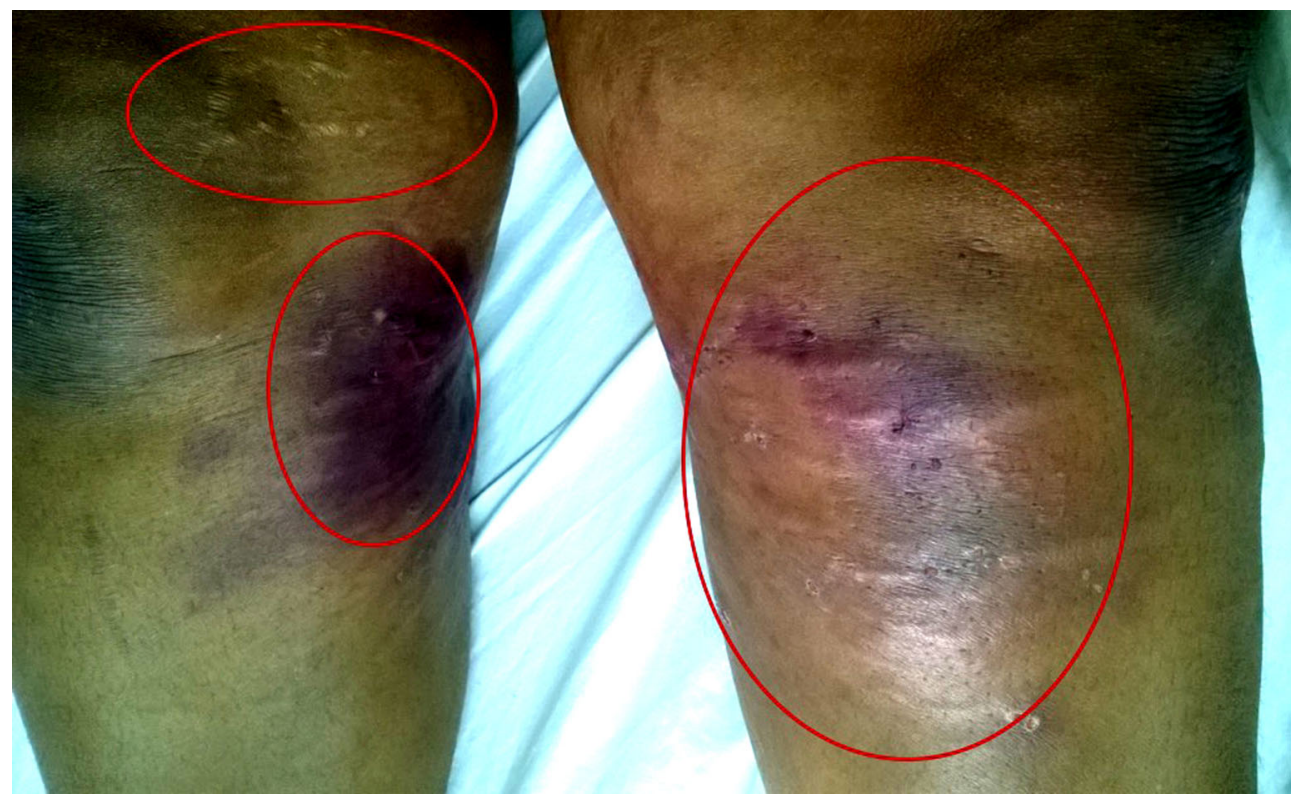

Fig. 3 Cordons papuleux érythémateux et serpigineux de la face antéro-interne des jambes / Erythematosus, serpiginous and papulous tracks of the antero-internal side of the legs

tropicales, dans le service de maladies infectieuses et tropicales de l'hôpital Pitié Salpêtrière (France) à 7/74 cas entre 2008 et $2013[1,18]$. En zone tropicale à Cotonou (Bénin), elle était de 3/46 cas en 2016 dans une série rétrospective hospitalière [4].

La majorité des cas de folliculite ankylostomienne concerne des jeunes adultes ayant visité des régions tropicales d'endémie $[2,10,3,18,21]$, alors que la forme typique de
LMCA est plus fréquente chez les enfants particulièrement en zone d'endémie $[4,6,14]$.

Sur le plan clinique, la folliculite ankylostomienne se manifeste sous forme de lésions papuleuses ou papulo-pustuleuses, particulièrement prurigineuses $[2,3,10,13,19,21]$. Les lésions sont nombreuses voire profuses, et localisées surtout aux fesses $[2,21]$. De ce fait, elle peut prêter à confusion avec d'autres dermatoses telles que les folliculites bactériennes, la scabiose 


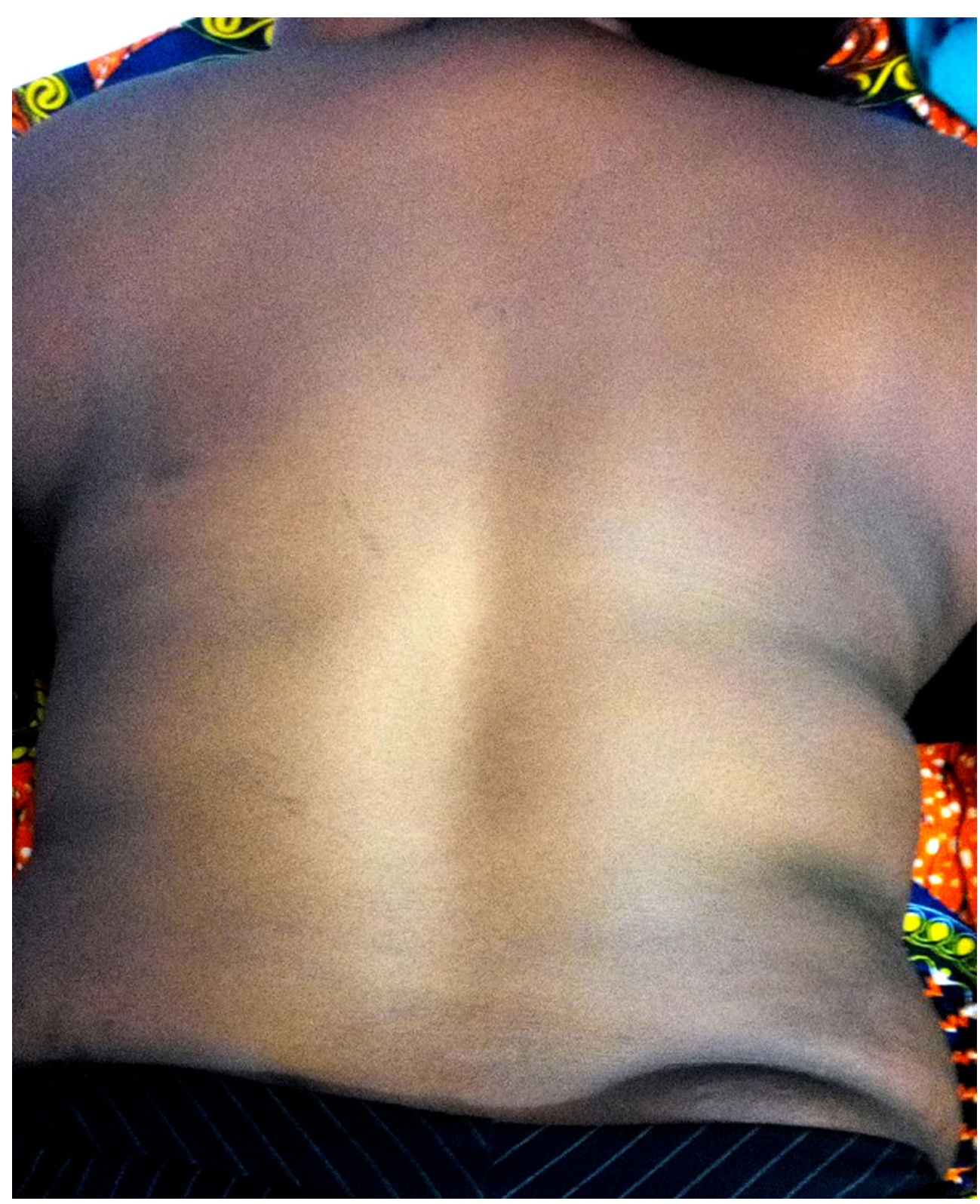

Fig. 4 Restitution de l'aspect normal de la peau 4 semaines après le traitement / Normal aspect of the skin after 4 weeks treatment

ou encore le prurigo aigu [2,6,21]. L'association à des lésions plus typiques de dermatite rampante permet le plus souvent d'aboutir au diagnostic $[3,4,6]$.

L'image histologique montre la larve enfermée dans le follicule pileux ou dans le derme ou dans l'épiderme ainsi qu'un infiltrat inflammatoire folliculaire et péri-vasculaire lympho-hystiocytaire et une prédominance d'éosinophiles $[2,3,10,13,19,21]$. Une étude récente de l'image ultrasonographique de LMCA a confirmé la présence de la larve dans le follicule pileux, voire dans le derme et l'hypoderme [11].

La durée d'incubation de la LMCA est très variable et se situe en moyenne autour de 5 jours à 1 mois. Cependant, des extrêmes ont été rapportés allant d'une heure à 7 mois. La variabilité de cette période d'incubation serait liée selon les auteurs aux facteurs intrinsèques à l'hôte et/ou à l'espèce d'ankylostome en cause [5-7,11,15].

La fragilisation de la barrière cutanée due à l'atrophie cutanée et l'immunodépression locale par les dermocorticoïdes contenus dans les produits dépigmentants pourraient expliquer en partie la survenue de la folliculite profuse chez notre patiente en 48 heures. L'association de cordons serpigineux typiques nous a permis d'éliminer les folliculites bactériennes et la scabiose assez fréquentes sur ces terrains [9].

L'albendazole et l'ivermectine utilisées dans les mêmes conditions que dans la forme typique se sont révélées moins efficaces $[8,17,20]$ : ceci a conduit à suggérer un traitement 
prolongé entre 3 et 7 jours avec l'albendazole ou la répétition de la prise de l'ivermectine $[3,12,20]$. L'albendazole a été efficace chez notre patiente après 7 jours successifs de traitement. Aucun effet secondaire n'a été noté.

\section{Conclusion}

La folliculite ankylostomienne est une forme rare de la LMCA qui suscite un intérêt en raison des risques d'errance diagnostique et de ses modalités thérapeutiques différentes de celles de la forme typique.

Liens d'intérêts Les auteurs déclarent ne pas avoir de liens d'intérêts.

\section{Références}

1. Caumes E, Carrière J, Guermonprez G, et al (1995) Dermatoses associated with travel to tropical countries: a prospective study of the diagnosis and management of 269 patients presenting to a tropical disease unit. Clin Infect Dis 0(3):542-8

2. Caumes E, Ly F, Bricaire F (2002) Cutaneous larva migrans with folliculitis: case report of seven cases and review of literature. $\mathrm{Br}$ J Dermatol; 146: 314-6

3. Clyti E, Caumes E, Deligny C, Sainte-Marie D, et al (2016) Folliculite ankylostomienne en Guyanne Française. Ann Dermatol Venereol 143(10):665-6. doi: 10.1016/j.annder.2016.05.002

4. Dégboé B, Agbéssi N, d'Almeïda C, Habib A, et al (2017) Larva migrans cutanée ankylostomienne: aspects épidémiologiques cliniques et thérapeutiques en Dermatologie à Cotonou de 2013 à 2016 au Bénin. Annales de l'Université de Parakou, Série « Sciences de la santé » 6(2):34-6

5. Feldmeier H, Schuster A (2012) Mini review: hookworm-related cutaneous larva migrans. Eur J Clin Microbiol Infect Dis 31 (6):915-8. doi: 10.1007/s10096-011-1404-x. Epub 2011 Sep 16.

6. Heukelbach J, Feldmeier H (2008) Epidemiological and clinical characteristics of hookworm-related cutaneous larva migrans. Lancet Infect Dis 8(5):302-9. doi: 10.1016/S1473-3099(08)70098-7

7. Hochedez P, Caumes E (2008) Common Skin Infections in Travelers. J Travel Med 15(4):252-62. doi: 10.1111/j.1708-8305. 2008.00206.x

8. Kincaid L, Klowak M, Klowak S, Boggild AK (2015) Management of imported cutaneous larva migrans: A case series and mini-review. Travel Med Infect Dis 13(5):382-7. doi: 10.1016/ j.tmaid.2015.07.007. Epub 2015 Jul 29
9. Kouotou EA, Zoung-Kanyi B, Fouda Nouind CC, et al (2015) Dépigmentation volontaire, pratique et dermatoses associées chez les commerçantes de Yaoundé (Cameroun). Ann Dermatol Venereol 142:443-5. doi: 10.1016/j.annder.2015.02.023

10. Miller AC, Walker J, Jaworski R, Launey W (1991) Hookworm Folliculitis. Arch Dermatol 127(4):547-9

11. Ogueta I, Navajas-Galimany L, Concha-Rogazy M, et al (2019) Very High- and High-Frequency Ultrasound Features of Cutaneous Larva Migrans. J Ultrasound Med 38(12):3349-58. doi: 10.1002/jum.15023. Epub 2019 May 20.

12. Quashie NB, Tsegah E (2015) An unusual recurrence of pruritic creeping eruption after treatment of cutaneous larva migrans in an adult Ghanaian male: a case report with a brief review of literature. Pan Afr Med J 21:285. doi: 10.11604/pamj.2015.21.285.5612. eCollection 2015

13. Rivera-Roig V, Sánchez JL, Hillyer GV (2008) Hookworm folliculitis. Int J Dermatol 47(3):246-8. doi: 10.1111/j.1365-4632. 2008.03469.x

14. Saka B, Kombaté K, Mouhari-Touré A, et al (2012) Larva migrans cutanée ankylostomienne en Dermatologie à Lomé, Togo, de 2006 à 2011. Ann Dermatol Venereol 139(8-9): 564-6. doi: 10.1016/ j.annder.2012.05.014

15. Siriez J-Y, Angoulvant F, Buffet P, et al (2010) Individual variability of the cutaneous larva migrans (CLM) incubation period. Pediatr Dermatol 27(2):211-2. doi: 10.1111/j.1525-1470.2010.01107.x.

16. Tianyi F-L, Agbor VN, Kadia BM, Dimala CA (2018) An unusual case of extensive truncal cutaneous larva migrans in a Cameroonian baby: a case report. J Med Case Rep 12(1):270. doi: 10.1186/s13256-018-1792-y

17. Vanhaecke C, Perignon A, Monsel G, et al (2014) The efficacy of single dose ivermectin in the treatment of hookworm related cutaneous larva migrans varies depending on the clinical presentation. J Eur Acad Dermatol Venereol 28(5):655-7. doi: 10.1111/ jdv.12097. Epub 2013 Feb 1

18. Vanhaecke C, Perignon A, Monsel G, et al (2014) Aetiologies of creeping eruption: 78 cases. Br J Dermatol 170(5):1166-9. doi: 10.1111/bjd.12637.

19. Veraldi S, Bottini S, Carrera C, Gianotti R (2005) Cutaneous larva migrans with folliculitis: a new clinical presentation of this infestation. J Eur Acad Dermatol Venereol 19(5): 628-30

20. Veraldi S, Bottini S, Rizzitelli G, Persico MC (2012) One-week therapy with oral albendazole in hookworm-related cutaneous larva migrans: A retrospective study on 78 patients. J Dermato$\log$ Treat 23(3):189-91. doi: 10.3109/09546634.2010.544707. Epub 2011 Feb 6

21. Veraldi S, Persico MC, Francia C, et al (2013). Follicular cutaneous larva migrans: a report of three cases and review of the literature. Int J Dermatol. 2013 Mar;52(3):327-30. doi: 10.1111/j.13654632.2012.05723.x 